\title{
Identification and Functional Analysis of LecRLK Genes in Taxodium 'Zhongshanshan'
}

\author{
Jinbo Guo ${ }^{1}$, Hao Duan ${ }^{1}$, Lei Xuan ${ }^{1}$, Ziyang Wang ${ }^{1}$, Jianfeng Hua $^{1}$, Chaoguang Yu ${ }^{1}$, Yunlong Yin $^{1}$, Mingzhi Li $^{2}$, \\ Ying Yang ${ }^{\text {Corresp. } 1}$ \\ 1 Jiangsu Engineerng Research Center for Taxodium Rich. Germplasm Innovation and Propagation, Institute of Botany, Jiangsu Province and Chinese \\ Academy of Sciences (Nanjing Botanical Garden Mem. Sun Yat-Sen), Nanjing, China \\ 2 \\ Genepioneer Biotechnologies Co. Ltd, Nanjing, China \\ Corresponding Author: Ying Yang \\ Email address: yingyang@cnbg.net
}

Background. Lectin receptor-like protein kinases (LecRLKs) can transform external stimuli into intracellular signals and play important regulatory roles in plant development and response to environmental stressors. However, research on the LecRLK gene family of conifers has seldom been reported.

Methods. Putative LecRLK genes were identified in the transcriptome of Taxodium 'Zhongshanshan.' The classification, domain structures, subcellular localization prediction, and expression patterns of LecRLK genes as well as co-expressed genes were analyzed using bioinformatics methods. Fifteen representative genes were further selected for qRT-PCR analysis in six tissues and under five different environmental stressor conditions.

Results. In total, 297 LecRLK genes were identified, including 155 G-type, 140 L-type, and 2 C-type. According to the classification, G-type and L-type LecRLK genes both can be organized into seven groups. The domain architecture of G-type proteins were more complex compared with that of L- and C-type proteins. Conservative motifs were found in G-type and L-type diverse lectin domains. Prediction and transient expression experiments to determine subcellular localization showed that LecRLKs were mainly concentrated in the cell membrane system, and some members were located at multiple sites at the same time. RNA-seq-based transcriptomics analysis suggested functional redundancy and divergence within each group. Unigenes co-expressed with LecRLKs in the transcriptome were found to be enriched in pathways related to signal transduction and environmental adaptation. Furthermore, qRT-PCR analysis of representative genes showed evidence of functional divergence between different groups.

Conclusions. This is the first study to conduct an identification and expression analysis of the LecRLK gene family in Taxodium. These results provide a basis for future studies on the evolution and function of this important gene family in Taxodium. 


\section{Identification and Functional Analysis of LecRLK Genes in Taxodium 'Zhongshanshan'}

Jinbo Guo ${ }^{1}$, Hao Duan ${ }^{1}$,Lei Xuan ${ }^{1}$, Ziyang Wang ${ }^{1}$, Jianfeng Hua ${ }^{1}$, Chaoguang Yu ${ }^{1}$, Yunlong Yin $^{1}$, Mingzhi $\mathrm{Li}^{2}$ and Ying Yang ${ }^{1 *}$

1 Jiangsu Engineerng Research Center for Taxodium Rich. Germplasm Innovation and Propagation, Institute of Botany, Jiangsu Province and Chinese Academy of Sciences (Nanjing Botanical Garden Mem. Sun Yat-Sen), Nanjing, 210042, China.

${ }^{2}$ Genepioneer Biotechnologies Co. Ltd, Nanjing, 210014, China

Corresponding Author:

Ying Yang ${ }^{1}$

Nanjing Botanical Garden Mem. Sun Yat-Sen, Nanjing, Jiangsu, 210014, China

Email address: yingyang@cnbg.net;

\section{Abstract}

Background. Lectin receptor-like protein kinases (LecRLKs) can transform external stimuli into intracellular signals and play important regulatory roles in plant development and response to environmental stressors. However, research on the LecRLK gene family of conifers has seldom been reported.

Methods. Putative LecRLK genes were identified in the transcriptome of Taxodium 'Zhongshanshan.' The classification, domain structures, subcellular localization prediction, and expression patterns of $L e c R L K$ genes as well as co-expressed genes were analyzed using bioinformatics methods. Fifteen representative genes were further selected for qRT-PCR analysis in six tissues and under five different environmental stressor conditions.

Results. In total, 297 LecRLK genes were identified, including 155 G-type, 140 L-type, and 2 Ctype. According to the classification, G-type and L-type LecRLK genes both can be organized into seven groups. The domain architecture of G-type proteins were more complex compared with that of L- and C-type proteins. Conservative motifs were found in G-type and L-type diverse lectin domains. Prediction and transient expression experiments to determine subcellular localization showed that LecRLKs were mainly concentrated in the cell membrane system, and some members were located at multiple sites at the same time. RNA-seq-based transcriptomics analysis suggested functional redundancy and divergence within each group. Unigenes coexpressed with LecRLKs in the transcriptome were found to be enriched in pathways related to signal transduction and environmental adaptation. Furthermore, qRT-PCR analysis of representative genes showed evidence of functional divergence between different groups. 
Conclusions. This is the first study to conduct an identification and expression analysis of the LecRLK gene family in Taxodium. These results provide a basis for future studies on the evolution and function of this important gene family in Taxodium.

\section{Introduction}

Throughout their life cycle, plants are exposed to constantly changing environments. The interaction between plant and environmental factors involves a series of signal perception and transduction pathways, including the receptor-like protein kinase (RLK) super family, which is a large cell-surface receptor family localized in the membrane(Walker 1994). RLKs are composed of three parts: (i) an N-terminus extracellular domain, (ii) a transmembrane domain, and (iii) a Cterminal intracellular serine/threonine kinase domain. RLK proteins are divided into 15 families based on their extracellular domains(Vaid et al. 2013). The lecRLK family is a group of RLKs containing extracellular lectin domains. Evidence acquired to date suggests that LecRLK family genes are present in many plant species and are thought to be plant-specific (Navarro-Gochicoa et al., 2003; Vaid et al., 2013). The transmembrane domain and intracellular kinase domain of LecRLKs are highly conserved, whereas the variability of the extracellular lectin domain is high. The LecRLK family can be divided into three subgroups based on the extracellular lectin domain: G-type, L-type, and C-type (Vaid et al., 2013). The G-type LecRLKs are proteins with an ectodomain that resembles the Galanthus nivalis agglutinin (GNA) mannose-binding motif (Teixeira et al., 2018). G-type LecRLKs were also called B-type LecRLKs, because GNA was first isolated from Galanthus nivalis bulbs, and B-lectin (PF01453) is the domain name of GNA in the Pfam database (Teixeira et al., 2018). The domain architecture of G-type LecRLK always contains a tandem repeat of a GNA (B-lectin) domain, an S-locus glycoprotein domain and/or a Pan/Apple (PNA) domain and/or a protein kinase domain (Eggermont et al., 2017). The L-type (legume-like) LecRLKs are present in high amounts in legumes (Eggermont et al. 2017). The domain architecture of L-type LecRLK includes a lectin-legB domain and/or a protein kinase domain (Neha et al. 2013). The extracellular lectin-legB domain contains a typical sandwich-fold structure in two complex topologies, suggesting that oligosaccharides may be the ligands (Neha et al. 2013). C-type (calcium-dependent) LecRLKs rely on $\mathrm{Ca}^{2+}$ to function. They are abundant in mammals and participate in pathogen recognition and immune response. However, C-type LecRLKs seldom exist in plants, with studies only finding one C-type LecRLK in Arabidopsis (Eggermont et al., 2017), rice, corn, Populus (Yang et al., 2016), and Eucalyptus, and two C-type LecRLKs in soybean (Liu et al., 2018) and wheat (Triticum aestivum) (Shumayla et al., 2016).

Plant LecRLKs can sense different external stimuli through the diverse extracellular domain and transform extracellular signals into intracellular signals through the intracellular kinase domain to facilitate the regulation of cell physiological and biochemical processes. The L-type lectin receptor kinase-V.5 (LecRK-V.5) gene of Arabidopsis negatively regulates stomatal immunity; overexpressing LecRK-V.5 led to early stomatal reopening, making Arabidopsis more sensitive to pathogen invasion (Marie et al., 2012). High levels of L-type lectin-like protein kinase 1 (AtLPK1) expression could promote seed germination and cotyledon greening under 
78 high salt stress. Additionally, expression of AtLPK1 is reported to be strongly induced by 79 abscisic acid (ABA), methyl jasmonate, salicylic acid, and stress treatments (Huang et al., 2013). 80 Sun et al. reported that ABA, salinity, and drought stress could all induce the expression of the 81 Glycine soja S-locus LecRLK gene (GSSRK) in soybean, and A. thaliana transformed with 82 GsSRK showed strong salt tolerance and high yield (Sun et al., 2013). In another study, the 83 overexpression of Pisum sativum LecRLK in tobacco was shown to reduce ionic and osmotic 84 components under salt stress so as to achieve salt-alkali tolerance (Vaid et al., 2015). L-type 85 LecRLKs characterized from Antarctic moss (Pohlia nutans; PnLecRLK1) are reported to 86 enhance the tolerance of $A$. thaliana to low temperatures and increase its sensitivity to ABA (Liu 87 88 89 90 91 92 et al., 2017). In addition to the above functions, LecRLKs are important in plant growth and development. LecRK IV.2 gene expression destroys pollen formation and leads to male sterility (Wan et al., 2008). The A. thaliana LecRK-al gene is highly expressed in cells around injured areas, and $A$. thaliana L-type LecRK-V.5 is presumed to play an important role in cell division (Riou et al., 2002). GSSRK was found to be involved in plant architecture (Sun et al., 2018), and the Gossypium hirsutum GhlecRK gene may play a role in fiber development (Zuo et al., 2004). The LecRLK family may play important roles in plant development, hormone regulation, and responses to biotic and abiotic stressors.

'Zhongshanshan' is the general term for the interspecific hybrid clones of bald cypress (Taxodium distichum) and Montezuma cypress (T. mucronatum). Several cultivars have been registered in China, such as 'Zhongshanshan 302' (Wang et al. 2016), 'Zhongshanshan 405 (Yu et al. 2016),' and 'Zhongshanshan 406' (Fan et al. 2018). Taxodium species are all well-known water-tolerant pioneer trees and also have moderate salt tolerance and are native swamp forest tree species in coastal, brackish locations in the southern United States (Allen et al., 2015). Taxodium species typically have long life spans, with many individuals found to have lived for more than 1,000-2,000 years, indicating that they have strong resistance to pests and diseases(Creech et al. 2011). Therefore, Taxodium species have strong tolerance to biotic and abiotic stressors (Yang et al., 2018). Taxodium 'Zhongshanshan' has been found to show strong adaptability in China, and is now widely used for wetland ecological restoration, coastal shelter forest construction, saline-alkali land afforestation, urban and rural afforestation, and farmland forest networks (Changxiao et al., 2005), offering great landscaping and ecological values. Recently, exploring the stress resistance of Taxodium at the molecular level has become a research focus (Yu et al., 2016; Fan et al., 2018; Wang et al., 2017; Qi et al., 2014). Because of their inability to move, plant can only passively withstand changes in the external environment and various stimuli. Therefore, receptors that can sense and specifically bind these stimuli, thus signaling molecules are very important for plants to adapt to environmental stressors. Study of the structure and function of the LecRLK gene family will be helpful to reveal the stress-related signal transduction network in Taxodium 'Zhongshanshan.'

At present, research on the $L e c R L K$ gene family in woody plants has only concentrated on a few tree species, like Poplar and Eucalyptus (Yang et al., 2016). Conifers are rarely reported due to the lack of genomic information. With the development of high-throughput sequencing 
118 technology, the transcriptome of Taxodium has been published (Qi et al., 2014; Yu et al., 2016; 119 Wang et al., 2019), which has laid a foundation for the identification of large gene families. Here, 120 we conducted a comprehensive bioinformatics analysis of ThzLecRLK gene family based on 121 transcriptome data and analyzed the expression of 15 representative LecRLKs in different tissues 122 and under various stressors. This study provides insight for functional predictions of many 123 members of the LecRLK gene family and provides a framework for further functional investigation of these genes.

125

\section{Materials and Methods}

\section{Identification of ThzLecRLK sequences}

129

130

131

132

133

134

135

136

137

138

139

140

141

142

143

144

145

146

147

148

149

150

151

152

153

154

155

156

157

The unigene sequences of Taxodium 'Zhongshanshan' were derived from the previously determined salinity stress transcriptome (Yu et al., 2016), short-term waterlogging transcriptome (Qi et al., 2014), and adventitious roots transcriptome (Wang et al., 2019). Hidden Markov Model (HMM) profiles (PF00069, PF01453, PF00139, PF00059), which correspond to kinase, G-type lectin, L-type lectin, and C-type lectin domains, respectively, were downloaded from the Pfam database (http://pfam.xfam.org/) (El-Gebali et al., 2019). We retrieved genes containing a kinase domain by running the hmmsearch program (HMMER 2.3.2) to search the kinase profile (PF00069) against the proteomic sequences. Within this set of hypothetical kinase proteins, we searched for G-type lectin, L-type lectin, and C-type lectin HMM profiles (PF01453, PF00139, PF00059) (E value cut-off $<1$ ). Sequences in which we identified a protein kinase domain (PF00069), along with either a G-type lectin (PF01453), an L-type lectin (PF00139), or a C-type lectin domain (PF00059) were selected. Pfam and NCBI CCD (Conserved Domain Database) (https://www.ncbi.nlm.nih.gov/cdd/) were further used to check their kinase domain and corresponding lectin domains to determine the putative lecRLKs. ThzLecRLK genes were finally confirmed after removing redundant sequences with $97 \%$ similarity between different database.

\section{Sequence alignment, classification, and subcellular localization Classification}

Multiple alignments of the nucleotide and amino acid sequences were performed using MAFFT v7 (Katoh \& Standley, 2013), and the auto alignment mode was selected. The classification of group and subgroups was constructed based on the sequences of ThzLecRLK proteins from A. thaliana and Taxodium 'Zhongshanshan' using a neighbor-joining (NJ) method with 1,000 bootstrap replicates. ClustalX2.1 was used to construct NJ tree, and MEGA7.0 software (http://www.megasoftware.net/) (Kumar et al., 2016)was used for beautification. C-type proteins were used as the outgroup for the subgroup classification of G-type and L-type lecRLKs.

The protein theoretical molecular weight and theoretical isoelectric point (pI) were predicted using compute $\mathrm{pI} / \mathrm{MW}$ (http://au.expasy.org/tools). The subcellular localization of PtAspAT proteins was predicted using the PSORT (https://psort.hgc.jp/) and CELLO programs (http://cello.life.nctu.edu.tw/). In addition, ThzlecRLK-C-type-2 and ThzlecRLK-L-type-86 were selected for a transient expression experiment. The coding DNA sequence (CDS) regions were inserted into a 16318-hGFP expression vector (Xian Chunfeng Biotechnology Co., Ltd). The

Peer) reviewing PDF | (2019:02:35361:1:1:NEW 27 Jun 2019) 
158

159

160

161

162

163

164

165

166

167

168

169

170

171

172

173

174

175

176

177

178

179

180

181

182

183

184

185

186

187

188

189

190

191

192

193

194

195

196

197

transient expression vectors (35S::ThzlecRLK-C-type-2-GFP and 35S::ThzlecRLK-L-type-86GFP) containing green-fluorescence protein (GFP) were transferred into the protoplast of $A$.

thaliana following the method of Yoo et al.(Yoo et al. 2007). The fluorescent signals were observed with a TCS SP8 DIVE Confocal laser scanning microscope (Leica, Germany).

\section{Analysis of the protein domain of ThzLecRLK sequences}

The Pfam 32.0 program (El-Gebali et al., 2019), NCBI CDD and TMHMM Server v. 2.0 (http://www.cbs.dtu.dk/services/ TMHMM/) were used to statistically identify conserved domain and transmembrane helices (TMhelix) in the complete amino acid sequences of ThzLecRLK proteins . The positions of all conserved domains and TM were marked and shown in a diagram based on their physical location. The sequences of the conserved domain were generated using the online Weblogo platform (http://weblogo.berkeley.edu/) (Crooks et al., 2004).

\section{Transcriptional profile analysis}

For ThzLecRLK gene expression analysis, RNA-seq data of different tissues and treatments of Taxodium 'Zhongshanshan' were obtained from the pre-laboratory development of the stress transcriptome (Yu et al., 2016) and short-term waterlogging transcriptome (Qi et al., 2014). The waterlogging stress related transcriptome analysis included four groups of data: the untreated root, the untreated stem, the root after one hour of soil waterlogging, and the stem after one hour of soil waterlogging. The salt stress related transcriptome analysis included root data under four treatments, namely, untreated root (T1), $100 \mathrm{mM} \mathrm{NaCl}$ treatment for $1 \mathrm{~h}$ (T2), $200 \mathrm{mM} \mathrm{NaCl}$ treatment for $1 \mathrm{~h}$ (T3), and $200 \mathrm{mM} \mathrm{NaCl}$ treatment for $24 \mathrm{~h}$ (T4). The level of gene expression was calculated by determining the number of expected fragments per kilobase of transcript per million mapped reads (FPKM). Heatmaps were generated using the heatmap package in $\mathrm{R}$ version 3.4.0 with $\log 2(\mathrm{FPKM}+1)$ value.

\section{Co-expression gene analysis}

The eight stress conditions mentioned above, i.e., four waterlogging and four salt stress transcriptomes, were analyzed to determine unigenes co-expressed with LecRLKs. All the unigenes with FPKM $\geq 1$ were included to calculate the correlation coefficient. The $\mathrm{R}$ package 'psych' was employed to determine the Pearson's correlation coefficient (PCC) values between ThzLecRLK unigenes and other unigenes. Correlation pairs were deemed statistically significant when the $|\mathrm{PCC}|$ was $>0.9$ and the corrected p-vale (FDR method) was $<0.05$. The co-expressed genes were used to analyzed the hypergeometric Fisher exact test $(\mathrm{P}<0.05)$ and Benjamini correction $(\mathrm{FDR}<0.05)$ to construct the map of enriched KEGG pathways. The transcription factors (TFs) in the co-expression genes were identified by searching the plant transcription factor database PlantTFDB 4.0 with all assembled unigenes (Jin et al., 2017).

\section{RNA extraction $q R T-P C R$ analysis}

In September 2018, the clones of one-year-old Taxodium 'Zhongshanshan 406' were taken, the roots were washed and put into water for hydroponic growth for a month. Then plants were treated with ABA $(1 \mathrm{mM}), \mathrm{SA}(1 \mathrm{mM})$, mannitol $(200 \mathrm{mM}), \mathrm{NaCl}(300 \mathrm{mM})$, and $4{ }^{\circ} \mathrm{C}$ treatment respectively. Shoots were collected from the seedlings subjected to all treatments at $6 \mathrm{~h}$. Shoos 
198

199

200

201

202

203

204

205

206

207

208

209

210

211

212

213

214

215

216

217

218

219

220

221

222

223

224

225

226

227

228

229

230

231

232

233

234

235

236

untreated with any stresses were taken as control. For organ-specific expression, Roots, stems, phloem, xylem and leaves were collected from clones of one-year-old Taxodium 'Zhongshanshan 406 ' and fruits were taken from mature clones of 'Zhongshanshan 406'. Root samples were taken as control. Three different plants as three biological replicates were randomly harvested. All collected samples were frozen in liquid nitrogen immediately after harvest and stored at $-80{ }^{\circ} \mathrm{C}$ until RNA extraction.

Total RNA was extracted using an RNeasy ${ }^{\circledR}$ Plant Mini Kit (QIAGEN, Shanghai, China). First-strand cDNA was synthesized using an HiScript II Q RT SuperMix for qRT-PCR (+g DNA wiper) (Vazyme Biotechnology Co., Ltd, Nanjing, China). Primers for qRT-PCR were designed using Primer software (https://www.genscript.com/tools/ pcr-primers-designer) (Supplemental Files 1). SYBR Green Reagents were used to detect the target sequence. PCR amplifications were performed in a StepOnePlus real-time thermal cycler (Thermo Fisher Scientific, San Jose, CA, USA) in a final volume of $20 \mu \mathrm{L}$ containing $2.0 \mu \mathrm{L}$ of cDNA, $0.4 \mu \mathrm{L}$ of each primer (200 $\mathrm{nM}), 7.2 \mu \mathrm{L}$ of sterile water, and $10 \mu \mathrm{L}(2 \times)$ of SYBR Green qRT-PCR Master Mix Kit (Bimake, Nanjing, China). Adenine phosphoribosyl transferase was used as the control to minimize variations in the cDNA template. Data represent three biological replicates and three technical replicates. The conditions for amplification were $10 \mathrm{~min}$ of denaturation at $95^{\circ} \mathrm{C}$, followed by 40 cycles of $95^{\circ} \mathrm{C}$ for $15 \mathrm{~s}, 60^{\circ} \mathrm{C}$ for $30 \mathrm{~s}$, and $72^{\circ} \mathrm{C}$ for $30 \mathrm{~s}$, after which a melt curve was produced at $60 \sim 95^{\circ} \mathrm{C}$. (Ma et al., 2018). The relative expression levels of genes were calculated using the $2^{-\Delta \Delta C t}$ method (Xia et al., 2014).

\section{Results}

\section{Identification and classification of LecRLKs in Taxodium 'Zhongshanshan'}

Using the unigene functional annotation database of the Taxodium 'Zhongshanshan' transcriptome, 1,331 unigenes were predicted to contain a protein kinase domain (PF00069). After removing incomplete open reading frame (ORF) sequences, 446 unigenes remained. Online SMART (http://SMART.embl-heidelberg.de) software was used to view the schematic map of protein structures, remove sequences without transmembrane domain (TM) and LecRLK domains. Ultimately, a total of 297 unigenes were obtained (Supplemental Files 2). All LecRLK proteins were classified into G-type, L-type, and C-type according to the structure of the extracellular lectin domain. In total, 155 G-type, 140 L-type, and two C-type LecRLKs were identified (Fig. 1, Supplemental Files 3).

\section{Classification of ThzLecRLKs}

A NJ tree was generated using multiple alignments of the complete LecRLK protein sequences of Taxodium 'Zhongshanshan' and A. thaliana. The cladograms of G-type and L-type LecRLKs were constructed separately and clustered into subgroups according to homologous relationships (Fig. 2). The cladogram of the 155 G-type members of the LecRLK family proteins of Taxodium 'Zhongshanshan' were divided into seven clusters. Some of these groups were then subdivided, based on distinct clade formation. Group II, IV and VII was subdivided into three 
237 subgroups (II-a to II-c, IV-a to IV-c, VII-a to VII-c), and Group VI was subdivided into five 238 subgroups (V-a to $\mathrm{V}$-e). Most AtLecRLKs were assigned to the same subgroup with only 239 At2G19130.1 being assigned to the Group VI-a subgroup and At4G32300.1 to the Group IV-a 240 clade (Fig. 2A, Supplemental Files 4). Similarly, for the L-type LecRLKs, the cladogram was 241 also divided into seven clusters. Group II was subdivided into seven subgroups (II-a to II-g), 242 Group V was subdivided into four subgroups (V-a to V-d), and Group VI was subdivided into 243 five subgroups (VI-a to VI-e). Each group, except the singleton Group V and VII, contained at 244 least one $A$. thaliana L-type LecRLK (Fig. 2B, Supplemental Files 5). The division of each 245 group was supported by high bootstrap values.

\section{Domain architecture analysis}

247 Predictive domain architecture and organization analysis assisted in the discovery and 248 characterization of the conserved domain of ThzLecRLKs (Fig. 3). The domain architecture of 249 G-type LecRKs were known to always have a tandem repeat of a GNA (B_lectin) domain, an S250 locus glycoprotein domain and/or a PNA domain and/or a protein kinase domain (Fig. 4A). Within the 155 G-type ThzLecRLKs, 87 contained all four basic domains (kinase, B-lectin, S-

253

254

255

256

257

258

259

260

261

262

263

264

265

266

267

268

269

270

271

272

273

274

275

276 locus glycoprotein, PAN), 25 contained kinase, B-lectin, and S-locus glycoprotein, 15 contained kinase, B-lectin and PAN, and the remaining 28 ThzLecRLKs only had kinase and B-lectin domains (Supplemental Files 6). In addition, an APH domain was found in the ThzLecRLK-Gtype-109 kinase domain, and a PIP49_C domain was identified in the ThzLecRLK-G-18 kinase domain. Compared with G-type proteins, the domain architectures of L- and C-type ThzLecRLKs were less complex. The L-type ThzLecRLK agglutinin domain contained only a lectin_legB domain and kinase domain (Fig. 4B). Both C-type ThzlecRLKs are composed of a lectin-C domain and kinase domain (Fig. 4C). All of the ThzLecRLKs were predicted to contain at least one TM domain, with 187 (G-type:96, L-type:90, C-type:1) ThzLecRLKs containing only one TM domain, 93 (G-type:43, L-type:49, C-type:1) containing two TM domains, and 17 (G-type:16, L-type:1) containing three TM domains.

To investigate the presence of conserved motifs in the main domains of ThzLecRLK, the amino acid sequences of intracellular kinase domains of all ThzLecRLKs, G-type ectodomains (B_lectin domain, S-locus glycoprotein domain and PNA domain), and L-type ectodomains (lectin_legB domain) were aligned. For the kinase domains, the alignment revealed the overall conservation of the ATP binding (consensus motif GxGxxGxV) and the catalytic sites (consensus motif HxDxKxxN) (marked with red boxes) (Supplemental Files 7) (Hanks \& Hunter, 1995). Two conversed motifs were identified in the S-locus glycoprotein domain, including a GYM element (65 G, 66 Y, $67 \mathrm{M})$ and a cysteine-rich (C-rich) motif. The C-rich motif was similar to that of the poplar EGF domain (Yang et al., 2016). Since the epidermal growth factor (EGF) domain was not predicted by the software used here, the C-rich motif could overlap the sequence of the $\mathrm{N}$-terminal region of the S-locus glycoprotein and the C-terminal region of EGF domains. Three highly conserved motifs were found in the PNA domain of Gtype ThzLecRLKs, including an FFS element (18 F, 19 F, 20 S), RYDVS element (59 R, 60 Y, $61 \mathrm{D}, 62 \mathrm{~V}, 63 \mathrm{~S}, 64 \mathrm{D})$, and another C-rich motif. The C-rich motif in the middle of the PNA 
277 domain was also found to be highly conserved in poplar (Yang et al., 2016), Arabidopsis, and 278 tomato G-type LecRLKs (Teixeira et al., 2018). Three elements (WQWSD, NxxSKRK, and 279 WxxLxxP) were found to be conversed in B-lectin domains. In addition, four conversed motifs 280 were found within the lectin-leg B domain of L-type PtLecRLKs.

281

282

283

284

285

286

287

288

289

290

291

292

293

294

295

296

297

298

299

300

301

302

303

304

305

306

307

308

309

310

311

312

313

314

315

\section{Physical and chemical properties and prediction of subcellular localization}

After determining the conserved domains of ThzLecRLKs, we predicted the physicochemical properties and subcellular localization of these proteins. The results showed that 297 ThzLecRLKs had a predicted ORF sequence length ranging from 136 to 910 amino acids, a molecular weight ranging from 15.24 to $101.24 \mathrm{kDa}$, and $\mathrm{pI}$ value ranging from 4.90 to 9.56 (Supplemental Files 2).

PSORT and CELLO were used for the subcellular localization prediction, and 266 ThzLecRLKs had common predicted localizations by both methods. Of these, 213 ThzLecRLKs were located in the plasma membrane and 28 were located at more than one site. The remaining 9 were located in the chloroplasts, 3 in the extracytoplasmic surface, 6 in the nucleus, 6 in the cytoplasm and 1 in the mitochondrion (Table 1; Supplemental Files 8). In addition, two genes were selected for an instantaneous expression experiment to further explore the of subcellular localization characteristics of the LecRLK family. Fluorescence signals of ThzlecRLK-C-type-2 were observed on the plasma membrane and nucleus (Fig. 5). Fluorescence signals of ThzlecRLK-L-type-86 were observed on the plasma membrane, chloroplast, and nucleus (Fig. 6).

\section{Transcriptome heat map analysis}

As the first attempt to provide insight into the potential function of ThzLecRLK genes, we analyzed the expression of the $297 \mathrm{ThzLecRLK}$ genes under salt and waterlogging stress by mining previously published transcriptome data of Taxodium 'Zhongshanshan' (Yu et al., 2016; Qi et al., 2014). Under salt stress, 241 ThzLecRLKs (G-type: 129; L-type: 111; C-type: 1) were not detected $(F P K M=0)$ in any sample. We generated a heat map of the 56 (G-type:26; L-type: 29; C-type: 1) expressed ThzLecRLK genes (FPKM $>0$ ) using the FPKM values (Fig. 7A). Many genes belonging to the same groups were clustered together on the heat map, such as G-type ThzLecRLKs from group I (ThzLecRLK-G-type-29, ThzLecRLK-G-type-30, and ThzLecRLK-Gtype-115) and group VI-c (ThzLecRLK-G-type-59 and ThzLecRLK-G-type-117), and group VII-b (ThzLecRLK-G-type-54 and ThzLecRLK-G-type-112), L-type ThzLecRLKs from group VI-d (ThzLecRLK-L-type-117, ThzLecRLK-L-type-18, and ThzLecRLK-G-type-31), group III (ThzLecRLK-L-type-90, and ThzLecRLK-L-type-93), and group II-e (ThzLecRLK-L-type-118 and ThzLecRLK-L-type-19).

11 Under waterlogging stress, 241 ThzLecRLKs (G-type: 130; L-type: 110; C-type: 1) were not detected in any sample. The expression of the remaining 56 ThzLecRLKs (FPKM $\geq 1)$ under waterlogging stress were analyzed (Fig. 7B). Genes from the same group, such as L-type ThzLecRLKs from group VI-d (ThzLecRLK-L-type-110 and ThzLecRLK-L-type-96), and group VI-a (ThzLecRLK-L-type-130 and ThzLecRLK-L-type-123) and G-type ThzLecRLKs from 
316 group IV-c (ThzLecRLK-G-type-80, and ThzLecRLK-L-type-98) were also clustered close 317 together on the heat map.

318 In total, 87 ThzLecRLKs (G-type:42; L-type: 43; C-type: 2) were expressed under the two 319 different stress conditions. Among them, 31 ThzLecRLKs (G-type:17; L-type: 13; C-type: 1) 320 were only expressed under salt stress and another 31 ThzLecRLKs (G-type:16; L-type: 14; C321 type: 1) were only expressed under waterlogging stress. The remaining, 25 ThzLecRLKs (G-type: 322 9; L-type: 16) were found to potentially be involved in both stress responses.

\section{Co-expression analysis}

324 More than 9,000 co-expressed genes of the 87 ThzLecRLKs with RKPM $>0$ were identified. 325 Among them, 230 transcription factors (TFs) were predicted to be co-expressed with 20 326

327

328

329

330

331

332

333

334

335

336

337

338

339

340

341

342

343

344

345

346

347

348

349

350

351

352

353

354

355

ThzLecRLKs (G-type 6, L-type 14), indicating that there may be regulatory relationships between these ThzLecRLKs and TFs. Interesting, the 20 ThzLecRLKs were among those expressed under both stress conditions. The co-expressed TFs were mainly MYB (28), ERF (19), $\mathrm{C}_{2} \mathrm{H}_{2}$ (19), bHLH (14), GRAS (10), and NAC (9) (Fig. 8). ThzLecRLK-L-type128 had the greatest number of TFs (82), followed by ThzLecRLK-L-type86 (52) and ThzLecRLK-L-type88 (46) (Supplemental Files 9).

The enrichment pathway $(\mathrm{P}<0.05)$ of co-expressed genes was analyzed using Kyoto Encyclopedia of Genes and Genomes (KEGG). The most enriched pathways included folding, sorting, and degradation, environmental information processing, signal transduction, environmental adaptation, and plant pathogen interaction (Fig. 9). These results indicated that the ThzLecRLK genes were closely related to signal transduction, stress response, and other biological pathways.

\section{Differential expression of LecRLKs in various tissues}

In order to determine whether diversity in structure led to varying expression patterns in tissues (i.e., roots, stems, xylem, phloem, leaves, and fruits), we selected 15 genes (G-type: 7; Ltype: 6; C-type: 2) to be subject to qRT-PCR. We selected one gene from each group of G-type and L-type as representative genes, and all of them were found to be stress-responsive genes revealed by the RNA-seq-based transcriptomics analysis (Table 2). Of the 15 selected ThzLecRLK genes, six (G-type:3 and L-type:3) showed peak expression levels in roots and four (G-type:1 and L-type:3) showed peak expression levels in phloem (Fig. 10). In addition, the Gtype genes ThzLecRLK-G-type-97 and ThzLecRLK-G-type-98 exhibited peak expression levels in the stem and xylem, respectively; however, ThzLecRLK-G-type-30 showed peak expression levels in leaves. The L-type gene ThzLecRLK-L-type-109 exhibited high levels of expression in both stem and phloem tissues. The expression of ThzLecRLK-C-type-1 was the highest in fruit and the lowest in the stem, while the expression of ThzLecRLK-C-type-2 was the highest in leaves and lowest in xylem.

\section{Differential expression of LecRLKs under a variety of stressors}

To investigate the potential roles that LecRLK genes may play in different stress response mechanisms of Taxodium 'Zhongshanshan', seedlings were treated with ABA, salicylic acid (SA), mannitol, $\mathrm{NaCl}$, and low temperature $\left(4^{\circ} \mathrm{C}\right)$ (Fig. 11). Of the 15 genes screened, ten (G-type:4, L-

Peer) reviewing PDF | (2019:02:35361:1:1:NEW 27 Jun 2019) 
356

357

358

359

360

361

362

363

364

365

366

367

368

369

370

371

372

373

374

375

376

377

378

379

380

381

382

383

384

385

386

387

388

389

390

391

392

393

394

395

type:4 and C-type:2) showed peak expressions at $4{ }^{\circ} \mathrm{C}$. In addition, ThzLecRLK-G-type-90 showed the highest relative expression under exposure to ABA, and ThzLecRLK-G-type-91 showed the highest relative expression under mannitol treatment. We also found that the relative expression of ThzLecRLK-G-type-101 gene under ABA, SA, mannitol, $\mathrm{NaCl}$, and $4^{\circ} \mathrm{C}$ treatments decreased significantly compared with that in the nutrient water treatment. As for the L-type genes, ThzLecRLK-L-type-87 showed the highest relative expression under $\mathrm{NaCl}$ treatment and ThzLecRLK-L-type-109 showed the highest relative expression under exposure to ABA. Of these differentially expressed genes, ThzLecRLK-G-type-30, ThzLecRLK-G-type-98, ThzLecRLK-Ltype-93, and ThzLecRLK-L-type-111 were significantly induced by low temperature stress and their relative expression levels were 5.7, 7.4, 6.3, and 19 times higher than in the controls, respectively.

\section{Discussion}

\section{Identification and classification of ThzLecRLKs}

LecRLK family proteins were found to be distributed in all plants, with the number ranging from 21 to 325 in Ma's research(Ma et al. 2018). However, there was no correlation between the number of lecRLK genes and the genome size of species(Ma et al. 2018). In this paper, 297 LecRLK genes were identified from the transcriptome of Taxodium 'Zhongshanshan'. As these proteins are heavily involved in plant growth, development, and stress tolerance, the large number of LecRLKs in Taxodium 'Zhongshanshan' may indicate that Taxodium have evolved a large number of LecRLKs to respond to a longer life cycle and greater likelihood of exposure to more diverse external stimuli (Liu et al., 2018). We found 140 L-type, 155 G-type, and two Ctype ThzLecRLKs. All embryonic plants were found containing three subgroups of lecRLKs (L, $\mathrm{G}$ and $\mathrm{C}$ ). The number of G-type lecRLK is more than that of L-type lecRLK in most species, except Arabidopsis, Capsella rubella(Ma et al. 2018), shrub and corn(Yang et al. 2016). Wan et al. (Wan et al., 2008) considered that cross-pollinated plants contains more G-type proteins than self-pollinated plants like $A$. thaliana; this may be due to the fact that G-type LecRLKs contain a special S-locus glycoprotein domain that regulates the self-incompatibility of pollen. There are generally 1-3 C-type LecRLKs in plants, indicating the relative stability of C-type LecRLKs in both gymnosperms and angiosperms. Finding at least one C-type in each species suggested the indispensable role of C-type LecRLKs in plants. The number of G-type ThzLecRLKs was similar to most reported plants(Ma et al. 2018), ranged from 100 to 200. The number of L-type ThzLecRLKs was much higher than other species, which were all reported to be less than 100 . Taken together, these results suggested that the expansion of the LecRLK gene family in Taxodium compared to angiosperms may related to the disproportionate expansion of L-type LecRLKs.

\section{Classification of ThzLecRLKs}

The classification of G-type ThzLecRLKs and AtLecRLKs indicated that 30 out of the 32 G-type AtLecRLKs were assigned to independent clusters. Similarly, for L-type, 33 out of the 42 L-type AtLecRLKs were clustered into independent branches. According to the NJ tree of G- 
396

397

398

399

400

401

402

403

404

405

406

407

408

409

410

411

412

413

414

415

416

417

418

419

420

421

422

423

424

425

426

427

428

429

430

431

432

433

434

435

type and L-type ThzLecRLKs and AtLecRLKs, most sequences from the two species are separate, with only a few sequences from Arabidopsis clustered together with the sequences of Taxodium 'Zhongshanshan.' This suggested that ThzLecRLKs may have different evolutionary functions from similar proteins in A. thaliana. The same trend has also been observed between other woody and herbaceous plants (Yang et al., 2016). Specifically, the LecRLKs between different woody species formed clades separate from each other (Yang et al., 2016). Therefore, it can be concluded that the LecRLK sequences of different species differ greatly.

\section{Conserved domain analysis}

Through the domain analysis, we found that there are more G-type ThzLecRLKs than Ltype and C-type proteins. It is speculated that G-type ThzLecRLKs may play more diverse roles in plant growth, development, and response to external stimuli. Notably, the majority of ThzLecRLKs (62.96\%) contained only one TM domain, 93 contained two TMs, and 17 contained three TM domains. Yang et al.'s findings were similar for poplar (Yang et al., 2016). This indicated that LecRLK, as an important membrane binding protein, may bind to the membrane system in a variety of ways. Besides, almost all the genes (16/17) containing three TMs were G-type, which further supports the complexity of the G-type structure.

In order to bind various substrates, the extracellular lectin domains of the LecRLK family are highly variable. However, it can be inferred that, except those responsible for substrate binding specificity, some regions of the ectodomains are conserved among different members of the same family. Consistent with this hypothesis, several highly-conserved motifs were identified within the ectodomains of both G-type and L-type ThzLecRLKs. Two C-rich motifs were identified in the C-terminal region of the EGF motif and the middle of the PAN domain of Gtype ThzLecRLKs, similar other species (Yang et al., 2016; Liu et al., 2018; Teixeira et al., 2018). The EGF domain is predicted to be involved in the formation of disulfide bonds and the PAN domain is believed to be involved in protein-protein and protein-carbohydrate interactions (Naithani et al., 2007). These conversed motifs of ThzLecRLKs may serve as potential sites of protein-protein interactions. Other conserved motifs may be essential sites for protein activity (Teixeira et al., 2018).

\section{Prediction of subcellular localization}

Understanding the cellular locations of LecRLK expression is conducive to the analyses of function. Therefore, we predicted the subcellular localization of 297 ThzLecRLKs. The localization results showed that most ThzLecRLKs were located on the plasma membrane, but some were located on chloroplast, vacuole, nucleus, cytoplasm, mitochondrion, extrocytoplasmic surface and endocytoplasmic reticulum. Of the eight loci, all are membranous organelles, except the cytoplasm and extrocytoplasmic surface. Plasma membrane, vacuole, endocytoplasmic reticulum are monolayer organelles, while chloroplast, nucleus and mitochondrion are double membrane organelles (Bigay and Antonny, 2012). Based on the results of the prediction of the subcellular localization and transient expression experiments, we speculated that the expression sites of ThzLecRLK family members as membrane binding proteins were not limited to the cell membrane but the entire cell membrane system. This may also be one of the reasons that 
436 ThzLecRLKs have more than one TM domain. In addition, ThzlecRLK-C-type-2 and 437 ThzlecRLK-L-type-86 both had multiple locations within the cell, indicating that a considerable 438 number of ThzLecRLKs might be expressed not only in one location, but also in the whole 439 membrane system.

440 Gene expression analysis

441 The patter of gene expression is often suggestive of a gene's biological function. RNA442 sequencing and qRT-PCR were combined to reveal the expression patterns of ThzLecRLKs. 443 Although 297 ThzLecRLKs were identified, the majority (70.71\%) of the genes showed limited 444 levels of expression under salt and waterlogging stress. This result was consistent with Populus, 445 in which 78 out of 231 PtLecRLKs had limited expression in 24 different samples collected 446 under control and treatment conditions (Yang et al., 2016). Thus, it can be concluded that 447 although there is a large number of LecRLKs, only some may exert biological functions under 448 different space-time conditions.

449 Some ThzLecRLK genes in the same group on phylogenetic tree were found to have a 450 similar expression pattern, suggesting possible functional redundancy of genes within a cluster. 451 For example, three tightly clustered genes (ThzLecRLK-G-type-29, ThzLecRLK-G-type-30, and 452 ThzLecRLK-G-type-115) from G-type Group III-b were also closely clustered on the salt stress 453 heat map. Conversely, some genes had different expression patterns from others in the same 454 cluster, suggesting functional divergence within a subfamily. For example, three genes 455 (ThzLecRLK-G-type-59, ThzLecRLK-G-type-117, and ThzLecRLK-G-type-54) from the G-type 456 Group V-c were significantly induced by salt stress, while the other five genes of the same group 457 were undetectable. qRT-PCR of 15 representative genes from different groups found that there 458 was no obvious regularity in the expression of these representative genes in different tissues and 459 under different stressors, which may be caused by their structural differences, suggesting 460 functional divergence between different clusters.

461 Both the RNA-sequencing and qRT-PCR analysis showed that partial LecRLK genes could 462 be up-regulated or down-regulated by multiple stressors. This is common for AtLecRLKs. For 463 example, L-type LecRK-VI.2/LecRKA4.1, which played a role in the ABA stress response during 464 seed germination (Jiang and Yu, 2009), had upregulated expression after infection with 465 Fusarium oxysporum (Zhu et al., 2013), and also contributed to resistance against Pseudomonas 466

467

468

469

470

471 syringae and P. carotovorum (Singh et al., 2012). Some ThzLecRLKs were found to be stressspecific. Thus, ThzLecRLK genes acted on both the specific signal transduction pathways of different stressors and the intersections of different stress signal transduction networks.

qRT-PCR analysis revealed that seven genes were most highly expressed in roots compared with other tissues. In poplar, besides flower bud tissue, the root was found to have the most tissue-specific expression of LecRLK genes (Yang et al., 2016). Expression may be high in the 472 roots because many of the major stressors plants suffer from are due to changes in soil conditions, 473 such as drought, waterlogging, salt, pathogens, etc., and roots are the first organs coming in 474 contact with these conditions. Twelve of the 15 representative genes had their peak expression 475 levels under low temperature stress. This may be due to the fact that samples (leaves and stems) 
476 used for stress analyses were collected from aboveground tissues. Cold stress signal can be

477 directly perceived by plant leaves and stems, while other stress signals are first sensed by roots 478 and then transferred to other tissues. Therefore, the LecRLK genes in the sample may sense cold 479 stress earlier.

480

481

\section{Conclusions}

482

483

In summary, 297 LecRLKs genes were identified for the first time in the transcriptome of Taxodium 'Zhongshanshan,' including 155 G-type, 140 L-type, and two C-type genes.

484

485

486

487

488

489

490

491

492

493

494

495

496

497

498

499

500

501

502

503

504

505

506

507

508

509

510

511

512

513

514

515

516

517

518 Evolutionary, structural, and expression analyses suggested divergence of ThzLecRLK groups and functional redundancy of the members in the same group. The results of this study shed light on the evolution and function of ThzLecRLK, and provide a framework for further functional investigation of these genes.

\section{Acknowledgements}

We thank International Science Editing (http://www.internationalscienceediting.com ) for editing this manuscript.

\section{References}

Allen JA, Chambers JL, and Mckinney DM. 2015. Intraspecific variation in the response of Taxodium distichum seedlings to salinity. Forest Ecology and Management 70:203-214. DOI:10.1016/0378-1127(94)90087-6

Bigay J, and Antonny B. 2012. Curvature, lipid packing, and electrostatics of membrane organelles: defining cellular territories in determining specificity. Developmental Cell 23(5):886895. DOI:10.1016/j.devcel.2012.10.009

Changxiao L, Zhangcheng Z, Yun L, Changxiao L, Zhangcheng Z, and Yun L. 2006. Effect of soil water change on photosynthetic characteristics of Taxodium distichum seedlings in the hydro-fluctuation belt of the Three Gorges Reservoir Area. Frontiers of Forestry in China 1(2):163-169. DOI:10.1007/s11461-006-0013-9

Creech D, Zhou L, Yunlong Y, and Eguiluz-Piedra T. 2011. Can Taxodium be improved. Arnoldia 69:11-20.

Crooks GE, Hon G, Chandonia JM, and Brenner SE. 2004. WebLogo: a sequence logo generator. Genome Research 14:1188-1190. DOI:10.1101/gr.849004

Eggermont L, Verstraeten B, and Van Damme EJ. 2017. Genome-wide screening for lectin motifs in Arabidopsis thaliana. The plant genome 10.DOI:10.3835/plantgenome2017.02.0010ElGebali S, Mistry J, Bateman A, Eddy SR, Luciani A, Potter SC, Qureshi M, Richardson LJ, Salazar GA, Smart A, Sonnhammer E, Hirsh L, Paladin L, Piovesan D, Tosatto S, and Finn RD. 2019. The Pfam protein families database in 2019. Nucleic Acids Research 47:D427-D432. DOI:10.1093/nar/gky995

Fan W, Yang Y, Wang Z, Yin Y, Yu C, Shi Q, Guo J, Xuan L, and Hua J. 2018. Molecular cloning and expression analysis of three ThERFs involved in the response to waterlogging stress of Taxodium 'Zhongshanshan406' , and subcellular localization of the gene products. Peerj 6:e4434.DOI:10.7717/peerj.4434

Hanks SK, and Hunter T. 1995. Protein kinases 6. The eukaryotic protein kinase superfamily: 
519 kinase (catalytic) domain structure and classification. Faseb Journal 9:576-596. 520 DOI:10.1096/fasebj.9.8.7768349

521 Huang P, Ju HW, Min JH, Zhang X, Kim SH, Yang KY, and Kim CS. 2013. Overexpression of 522 L-type lectin-like protein kinase 1 confers pathogen resistance and regulates salinity response in 523 Arabidopsis thaliana. Plant Science 203-204:98-106. DOI:10.1016/j.plantsci.2012.12.019 Jiang W, and Yu D. 2009. Arabidopsis WRKY2 transcription factor mediates seed germination and postgermination arrest of development by abscisic acid. BMC Plant Biology 9(1):96. DOI:10.1186/1471-2229-9-96

Jin J, Tian F, Yang DC, Meng YQ, Kong L, Luo J, and Gao G. 2017. Plant TFDB 4.0: toward a central hub for transcription factors and regulatory interactions in plants. Nucleic Acids Research 45:D1040-D1045. DOI:10.1093/nar/gkw982

531 Katoh K, and Standley DM. 2013. MAFFT multiple sequence alignment software version 7: improvements in performance and usability. Molecular Biology And Evolution 30:772-780.

532 DOI:10.1093/molbev/mst010

534 Kumar S, Stecher G, and Tamura K. 2016. MEGA7: Molecular evolutionary genetics analysis Version 7.0 for Bigger Datasets. Molecular Biology and Evolution 33:1870-1874. DOI:10.1093/molbev/msw054

536

537 Liu P, Huang Y, Shi P, Yu M, Xie J, and Xie L. 2018. Duplication and diversification of lectin receptor-like kinases (LecRLK) genes in soybean. Scientific Reports 8(1):5861. DOI:10.1038/s41598-018-24266-6

539 Liu S, Wang J, Chen K, Zhang Z, and Zhang P. 2017. The L-type lectin receptor-like kinase 540 (PnLecRLK1) from the Antarctic moss Pohlia nutans enhances chilling-stress tolerance and 541 abscisic acid sensitivity in Arabidopsis. Plant Growth Regulation 81(3):409-418. 542 DOI:10.1007/s10725-016-0217-4

543 Ma N, Liu C, Li H, Wang J, Zhang B, Lin J, and Chang Y. 2018. Genome-wide identification of 544 lectin receptor kinases in pear: Functional characterization of the L-type LecRLK gene PbLRK138. Gene 661. DOI:10.1016/j.gene.2018.03.077 Marie DT, Dominique A, Ting-Yu H, Grace Jui-Chih L, Wei-Yen C, Yi-Chia L, and Laurent Z. 2012. The Arabidopsis lectin receptor kinase LecRK-V.5 represses stomatal immunity induced by Pseudomonas syringae pv. tomato DC3000. PLOS Pathogens 8(2) 8:e1002513. DOI:10.1371/journal.ppat.1002513

Neha, Vaid, Macovei, Narendra, and Tuteja. 2013. Knights in action: lectin receptor-like kinases in plant development and stress responses. Molecular Plant 6:1405-1418. DOI: $10.1093 / \mathrm{mp} / \mathrm{sst} 033$

553 Naithani S, Chookajorn T, Ripoll DR, and Nasrallah JB. 2007. Structural modules for receptor 554 dimerization in the S-locus receptor kinase extracellular domain. Proceedings of the National Academy of Sciences 104(29):12211-12216. DOI:10.1073/pnas.0705186104

Navarro-Gochicoa MT, Camut S, Timmers AC, Niebel A, Herve C, Boutet E, Bono JJ, Imberty A, and Cullimore JV. 2003. Characterization of four lectin-like receptor kinases expressed in roots of Medicago truncatula. Structure, location, regulation of expression, and potential role in the symbiosis with Sinorhizobium meliloti. Plant Physiology 133:1893-1910. DOI:10.1104/pp.103.027680

562

Qi B, Yang Y, Yin Y, Xu M, and Li H. 2014. De novo sequencing, assembly, and analysis of the Taxodium 'Zhongshansa' roots and shoots transcriptome in response to short-term waterlogging. BMC Plant Biology 14:201. DOI:10.1186/s12870-014-0201-y

564 Riou C, Hervé C, Pacquit V, Dabos P, and Lescure B. 2002. Expression of an Arabidopsis lectin 
565 kinase receptor gene, lecRK-a1, is induced during senescence, wounding and in response to 566 oligogalacturonic acids. Plant Physiology and Biochemistry 40:431-438. DOI:10.1016/s0981567 9428(02)01390-6

568 Shumayla Sharma S, Pandey AK, Singh K, and Upadhyay SK. 2016. Molecular characterization 569 and global expression analysis of lectin receptor kinases in bread wheat (Triticum aestivum). 570 PLoS One 11:e153925. DOI:10.1371/journal.pone.0153925

571 Singh P, Kuo YC, Mishra S, Tsai CH, Chien CC, Chen CW, Desclos-Theveniau M, Chu PW, 572 Schulze B, Chinchilla D, Boller T, and Zimmerli L. 2012. The lectin receptor kinase-VI.2 is 573 required for priming and positively regulates Arabidopsis pattern-triggered immunity. Plant Cell 574 24:1256-1270. DOI:10.1105/tpc.112.095778

575 Sun M, Qian X, Chen C, Cheng S, Jia B, Zhu Y, and Sun X. 2018. Ectopic expression of GsSRK 576 in Medicago sativa reveals its involvement in plant architecture and salt stress responses. 577 Frontiers in Plant Science 9:226. DOI:10.3389/fpls.2018.00226

578 Sun XL, Yu QY, Tang LL, Ji W, Bai X, Cai H, Liu XF, Ding XD, and Zhu YM. 2013. GsSRK, a 579 G-type lectin S-receptor-like serine/threonine protein kinase, is a positive regulator of plant 580 tolerance to salt stress. Journal of Plant Physiology 170:505-515. 581 DOI:10.1016/j.jplph.2012.11.017

582 Teixeira MA, Rajewski A, He J, Castaneda OG, Litt A, and Kaloshian I. 2018. Classification and 583 phylogenetic analyses of the Arabidopsis and tomato G-type lectin receptor kinases. BMC 584 Genomics 19:239. DOI:10.1186/s12864-018-4606-0

585 Vaid N, Macovei A, and Tuteja N. 2013. Knights in action: lectin receptor-like kinases in plant 586 development and stress responses. Molecular Plant 6:1405-1418. DOI:10.1093/mp/sst033

587 Vaid N, Pandey P, Srivastava VK, and Tuteja N. 2015. Pea lectin receptor-like kinase functions 588 in salinity adaptation without yield penalty, by alleviating osmotic and ionic stresses and 589 upregulating stress-responsive genes. Plant Molecular Biology 88:193-206. 590 DOI:10.1007/s11103-015-0319-9

591 Wang Z, Cheng Y, Yin Y, Yu C, Yang Y, Shi Q, Hao Z, and Li H. 2016. Genetic linkage map 592 construction and QTL mapping of seedling height, basal diameter and crown width of 593 Taxodium 'Zhongshanshan 302' $\times$ T. mucronatum. Springerplus 5:936. DOI:10.1186/s40064-016594 2617-3

595 Wang Z, Hua J, Yin Y, Gu C, Yu C, Shi Q, Guo J, Xuan L, and Yu F. 2019. An integrated 596 transcriptome and proteome analysis reveals putative regulators of adventitious root formation in 597 Taxodium 'Zhongshanshan'. International Journal of Molecular Sciences 20, 1225. 598 DOI:10.3390/ijms20051225

599 Walker JC. 1994. Structure and function of the receptor-like protein kinases of higher plants. 600 Plant Molecular Biology 26:1599-1609. DOI:10.1007/BF00016492

601 Wan J, Patel A, Mathieu M, Kim SY, Xu D, and Stacey G. 2008. A lectin receptor-like kinase is 602 required for pollen development in Arabidopsis. Plant Molecular Biology 67:469-482. 603 DOI:10.1007/s11103-008-9332-6

604 Wang Z, Yin Y, Hua J, Fan W, Yu C, Xuan L, and Yu F. 2017. Cloning and characterization of 605 ThSHRs and ThSCR transcription factors in Taxodium hybrid 'Zhongshanshan 406'. Genes (Basel) 606 8(7). DOI:10.3390/genes8070185

607 Yoo SD, Cho YH, and Sheen J. 2007. Arabidopsis mesophyll protoplasts: a versatile cell system 608 for transient gene expression analysis. Nature Protocols 2:1565-1572.

609 Yang Y, Labbé J, Muchero W, Yang X, Jawdy SS, Kennedy M, Johnson J, Sreedasyam A, 610 Schmutz J, Tuskan GA, and Chen J. 2016. Genome-wide analysis of lectin receptor-like kinases 
611 in Populus. BMC Genomics 17(1):699. DOI:10.1186/s12864-016-3026-2

612 Yang Y, Xuan L, Yu C, Wang Z, Xu J, Fan W, Guo J, and Yin Y. 2018. High-density genetic 613 map construction and quantitative trait loci identification for growth traits in (Taxodium 614 distichum var. distichum x T. mucronatum) x T. mucronatum. BMC Plant Biology 18:263. 615 DOI:10.1186/s12870-018-1493-0

$616 \mathrm{Yu} \mathrm{C}, \mathrm{Xu} \mathrm{S}$, and Yin Y. 2016. Transcriptome analysis of the Taxodium 'Zhongshanshan 405' 617 roots in response to salinity stress. Plant Physiology and Biochemistry 100:156-165. 618 DOI:10.1016/j.plaphy.2016.01.009

619 Zhu QH, Stephen S, Kazan K, Jin G, Fan L, Taylor J, Dennis ES, Helliwell CA, and Wang MB. 620 2013. Characterization of the defense transcriptome responsive to Fusarium oxysporum-infection 621 in Arabidopsis using RNA-seq. Gene 512:259-266. DOI:10.1016/j.gene.2012.10.036

622 Zuo K, Zhao J, Wang J, Sun X, and Tang K. 2004. Molecular cloning and characterization of 623 GhlecRK, a novel kinase gene with lectin-like domain from Gossypium hirsutum. DNA Seq 624 15:58-65. DOI:10.1080/1042517042000191454 
Figure 1 (on next page)

Classification of 297 LecRLKs genes.

The cladogram was constructed by neighbor-joining (NJ) method. Note that three different types of ThzLecRLKs are categorized clearly in three different clades (blue: G-type; Cyan: Ctype; Fuchsia: L-type) 







\section{Figure 2}

Classification of different groups of $\mathrm{G}$ and L-type ThzLecRLKs.

Different color regions are used to distinguish different subgroups. (A) Cladogram of $G$ type LecRLK gene family. Neighbor-joining (NJ) method was used to analyze the evolutionary trees of $155 \mathrm{G}$ Thzlecrks and $32 \mathrm{G}$ AtLecRLK. (B) Cladogram of $\mathrm{L}$ type LecRLK gene family. The cladogram of L-type ThzLecRLK analyzed 50 L-type ThzLecRLK and 39 AtLecRLK with NJ.
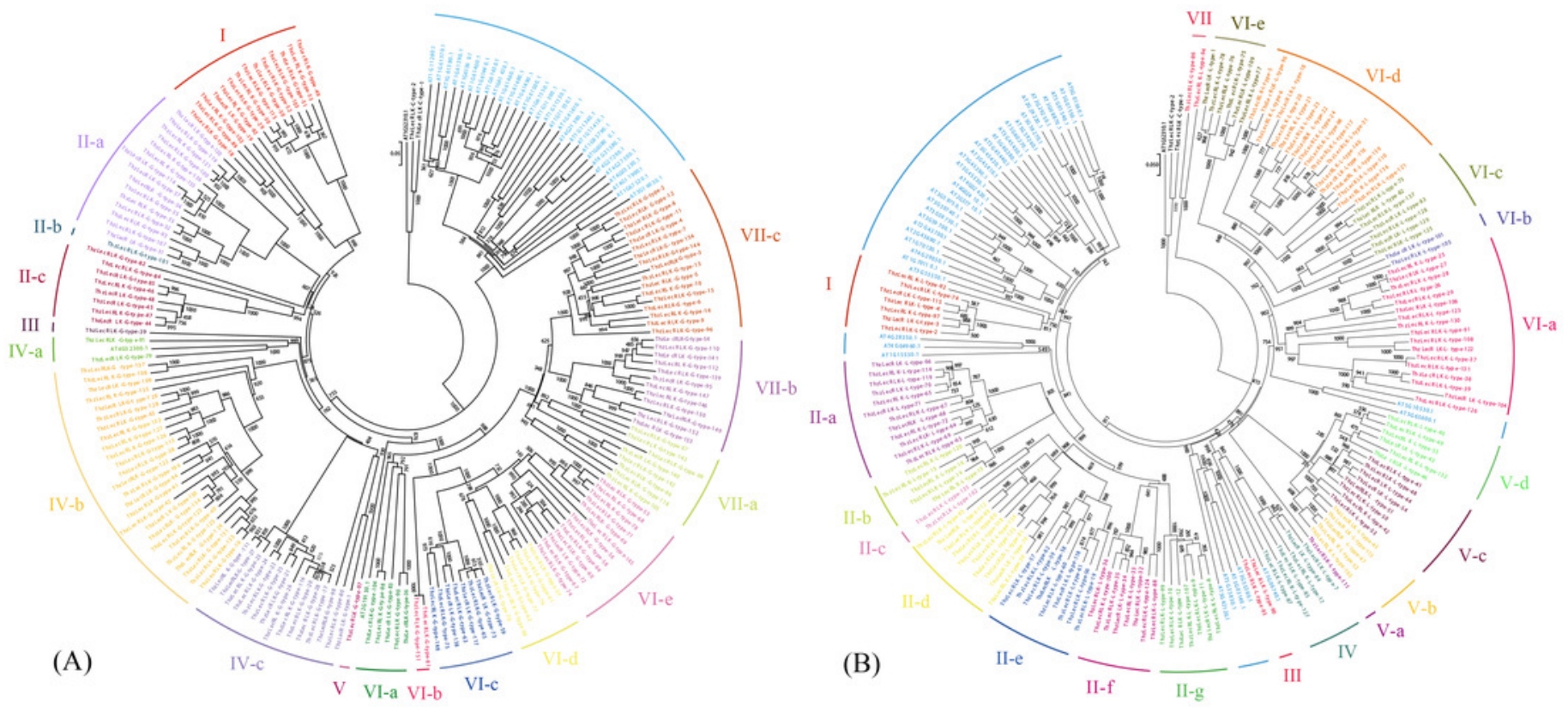
Figure 3

Conserved domain in lectin domain of ThzLecRLK (total 297) proteins.

C-type: conserved domain in lectin domain of C-type ThzLecRLKs (total two) protein; L-type: conserved domain in lectin domain of L-type ThzLecRLKs (total 140) protein; G-type: conserved domain in lectin domain of G-type LecRLKs (total 155) protein. 

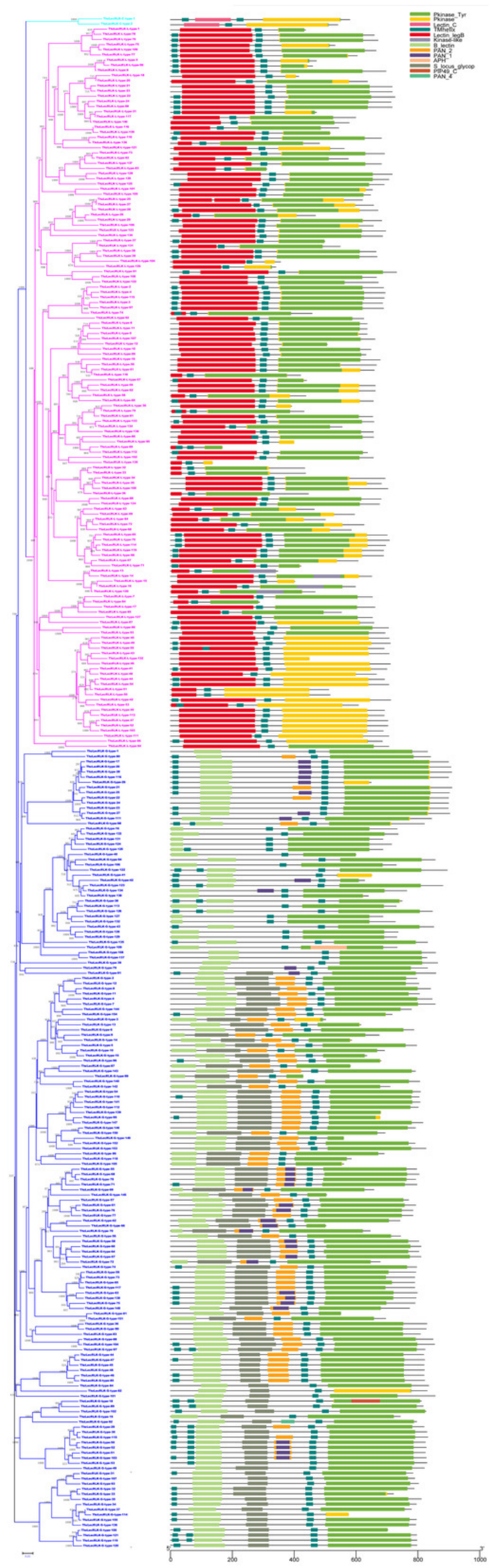

Peer) reviewing PDF | (2019:02:35361:1:1:NEW 27 Jun 2019) 


\section{Figure 4}

Line Model of the Three LecRLK Classes.

The LecRLK family can be divided into three subgroups based on the extracellular lectin domain: G-type, L-type, and C-type. (A)The domain architecture of L-type LecRLK includes a lectin-legB domain and/or a protein kinase domain(Pkinase). (B) The domain architecture of G-type LecRLK always contains a tandem repeat of a GNA (B-lectin) domain, an S-locus glycoprotein domain and/or a Pan/Apple (PNA) domain and/or a protein kinase domain. (C) Ctype LecRLKs characterized by the presence of calcium-dependent carbohydrate-binding domains.

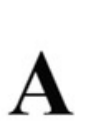

\section{B lectin}

\section{Pkinase}

B
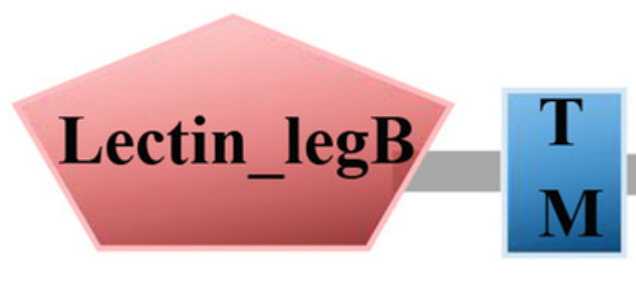

\section{Pkinase}

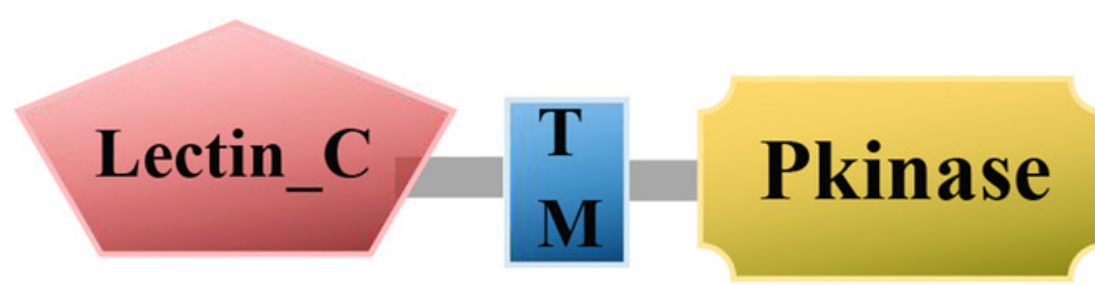




\section{Figure 5}

Subcellular localization of ThzLecRLK-C-type-2 in Arabidopsis thaliana.

A: Bright; B: DAPI, the protoplasts are stained with DAPI to visualize the nucleus; C: Chlo, Chloroplast autofluorescence (type2 Dil staining); D: GFP, fluorescence of ThzLecRLK-Ltype-86-GFP; E: GFP+DAPI, merged images of GFP and DAPI ones; F: GFP+Chlo, merged images of GFP and Chlo ones.
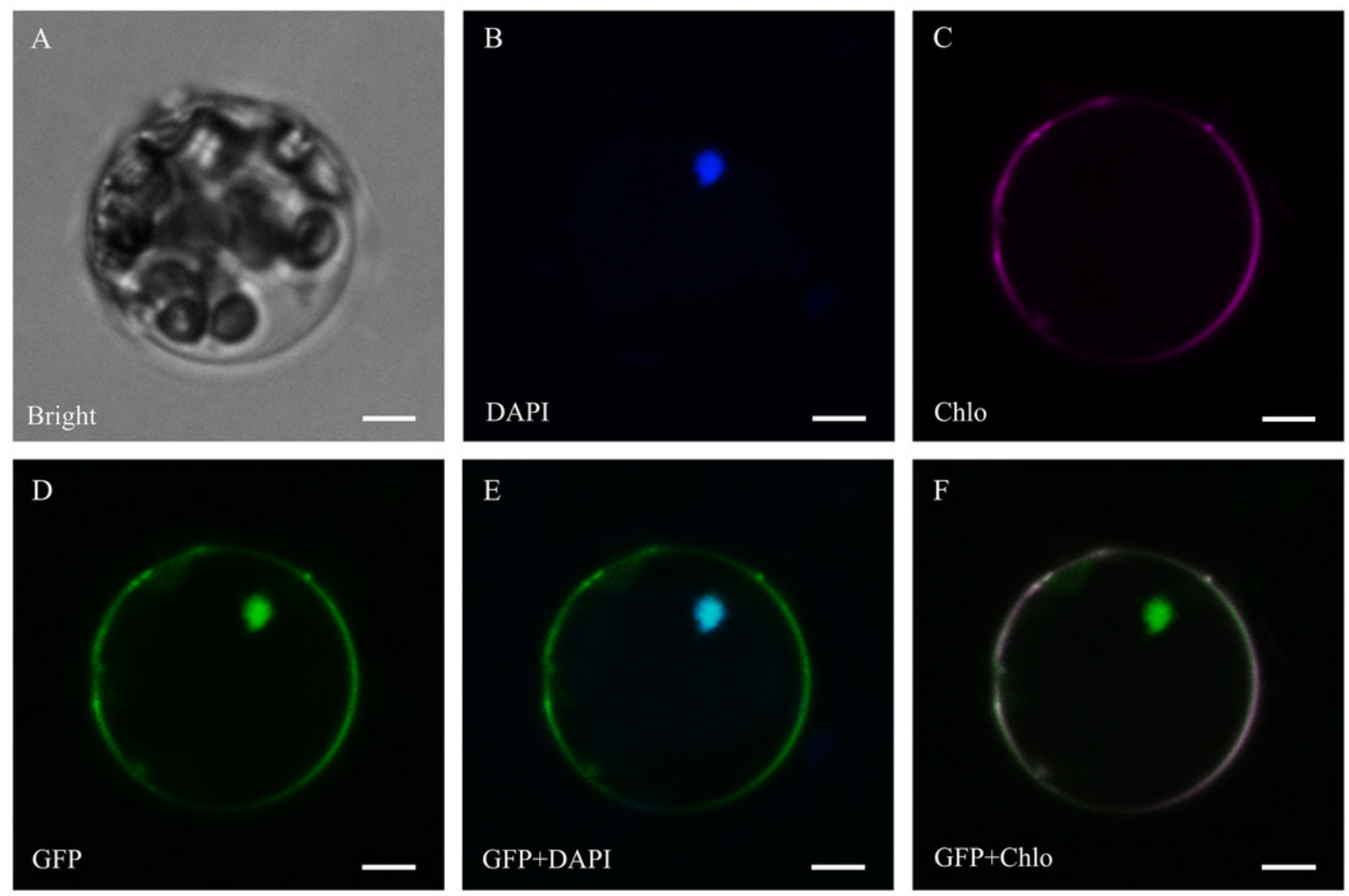


\section{Figure 6}

Subcellular localization of ThzLecRLK-L-type-86 in Arabidopsis thaliana.

A: Bright; B: DAPI, the protoplasts are stained with DAPI to visualize the nucleus; C:Chlo, Chloroplast autofluorescence; D:GFP, fluorescence of ThzLecRLK-L-type-86-GFP; E:GFP+DAPI, merged images of GFP and DAPI ones; F:GFP+Chlo, merged images of GFP and Chlo ones.
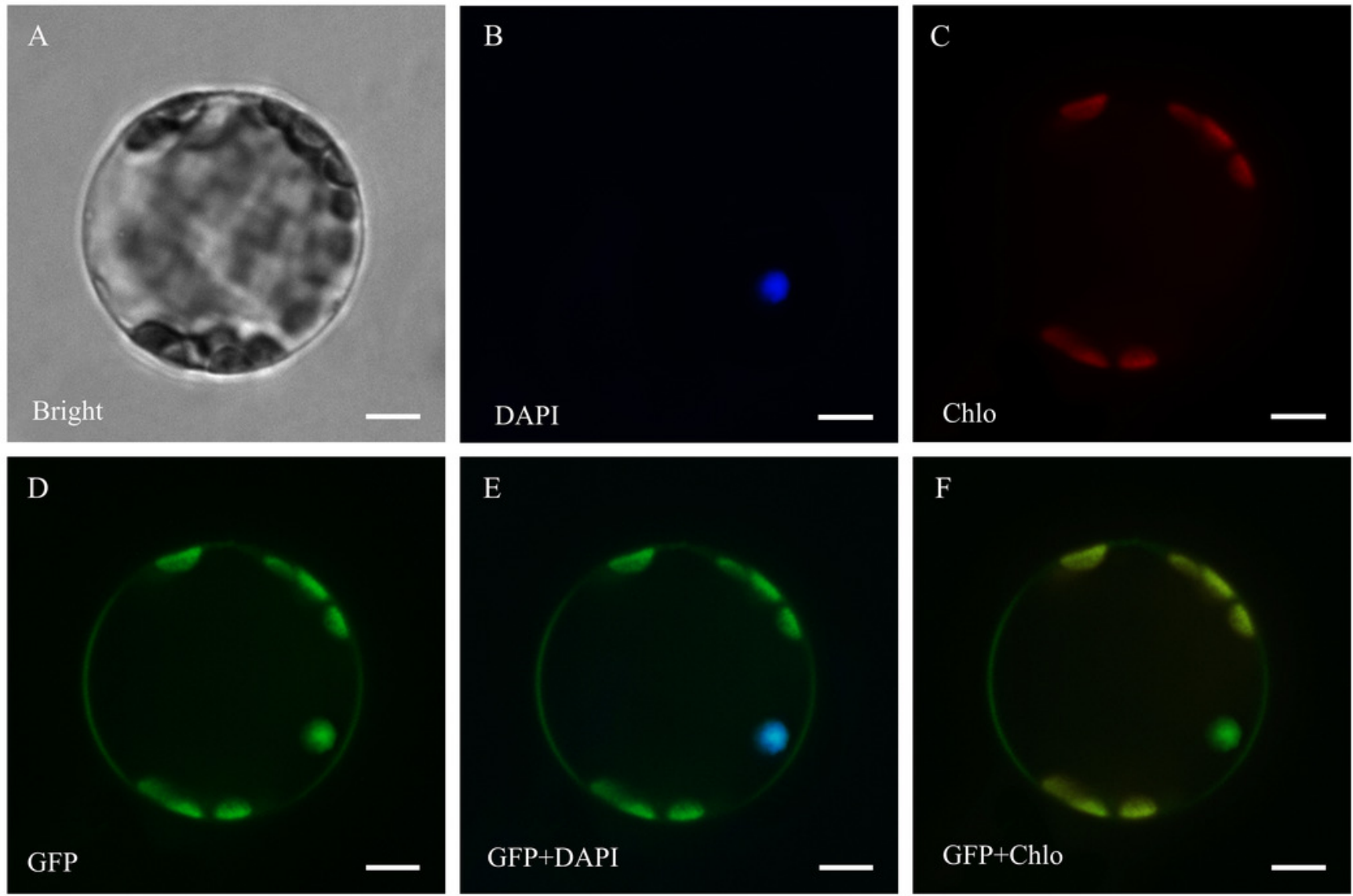


\section{Figure 7}

Expression patterns of ThzLecRLK genes.

RNA-seq data of different tissues and treatments of T. hybrid 'Zhongshanshan' were obtained from NCBI SRA (Sequence Read Archive). The specificity of gene expression was determined by comparing FPKM values in specific treatments. The classifications of genes in phylogenetic trees were listed next to the gene name. (A) The FPKM value of four different Nacl treatment including T1 (Untreated), T2 (Roots treated with $100 \mathrm{mM}$ Nacl for 1 hour), T3 (Roots treated with $200 \mathrm{mM} \mathrm{Nacl}$ for 1 hour) and T4 (Roots treated with $200 \mathrm{mM} \mathrm{Nacl}$ for 24 hour) were analyzed. (B) The FPKM value of four different waterlogging treatment including R1 (Untreated root), R2 (Root after 1 hour of waterlogging), S1 (Untreated leaves) and S2 (Root after 1 hour of leaves) were analyzed. 


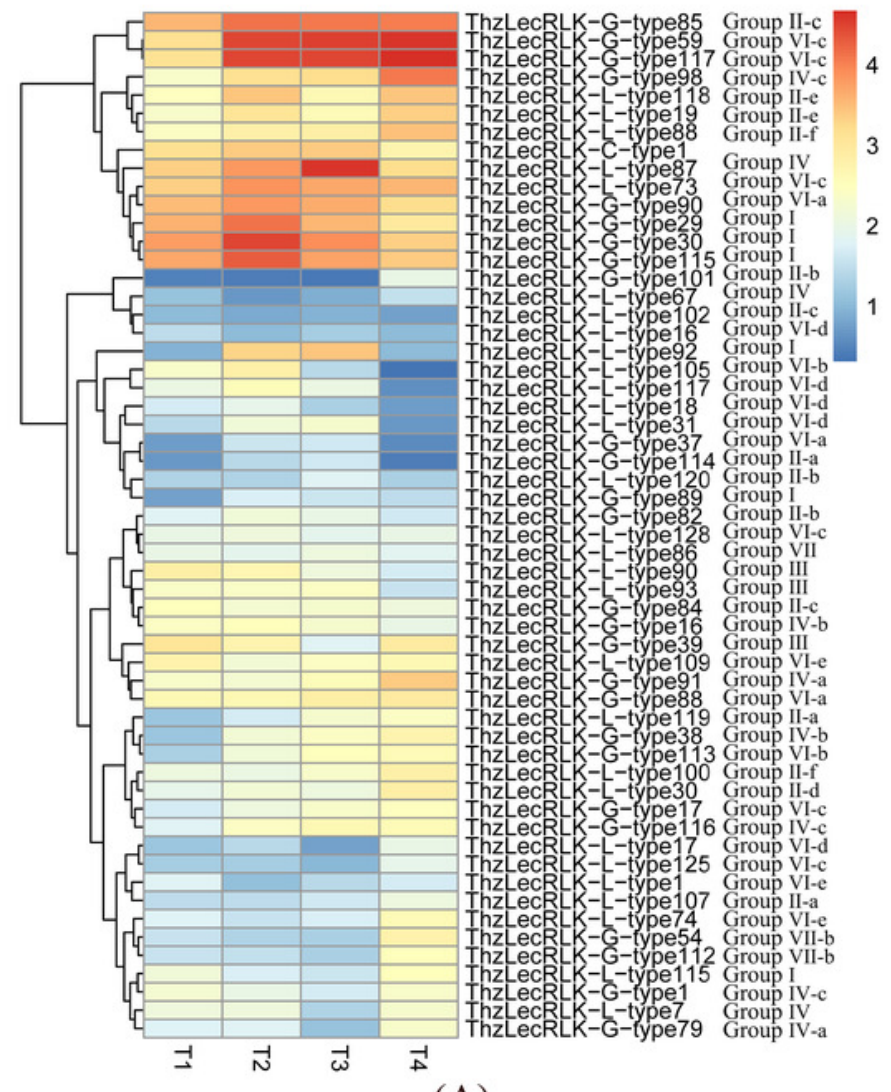

(A)

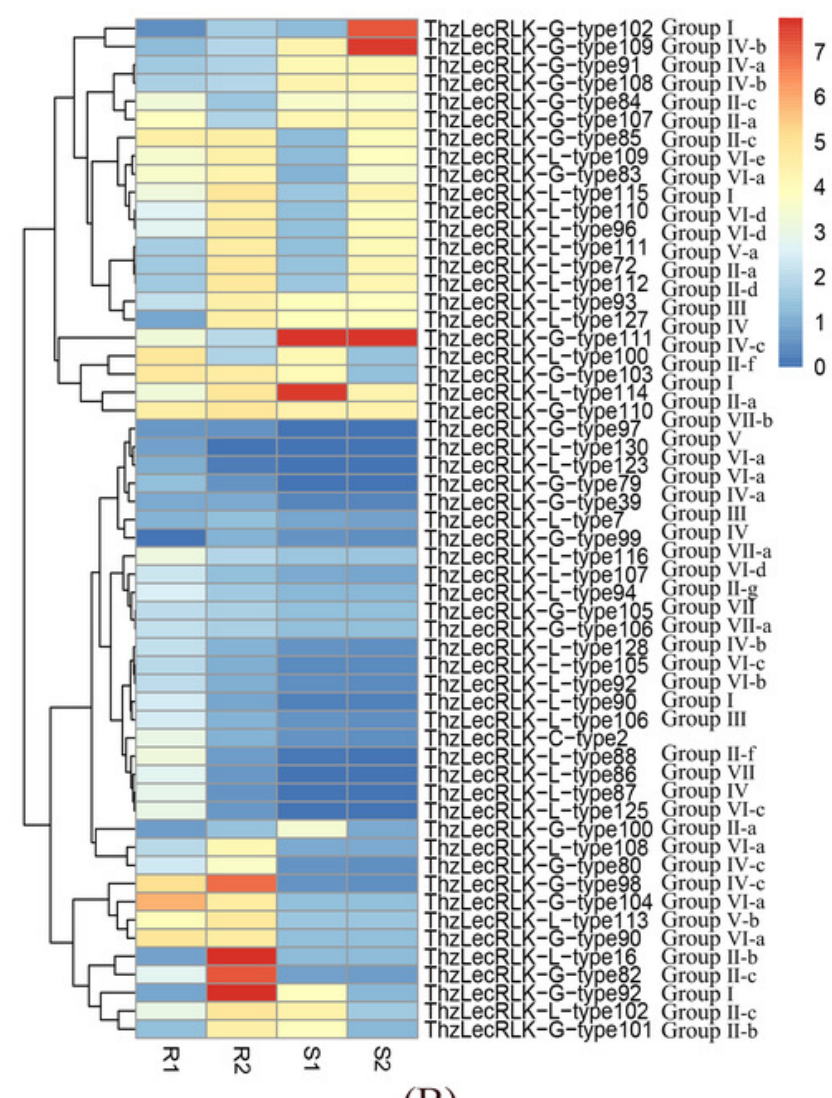

(B) 


\section{Figure 8}

Statistics of the number of transcription factors predicted to be co-expressed with ThzLeCRLKs.

The number of each type was listed next to the abbreviation of the transcription factor.

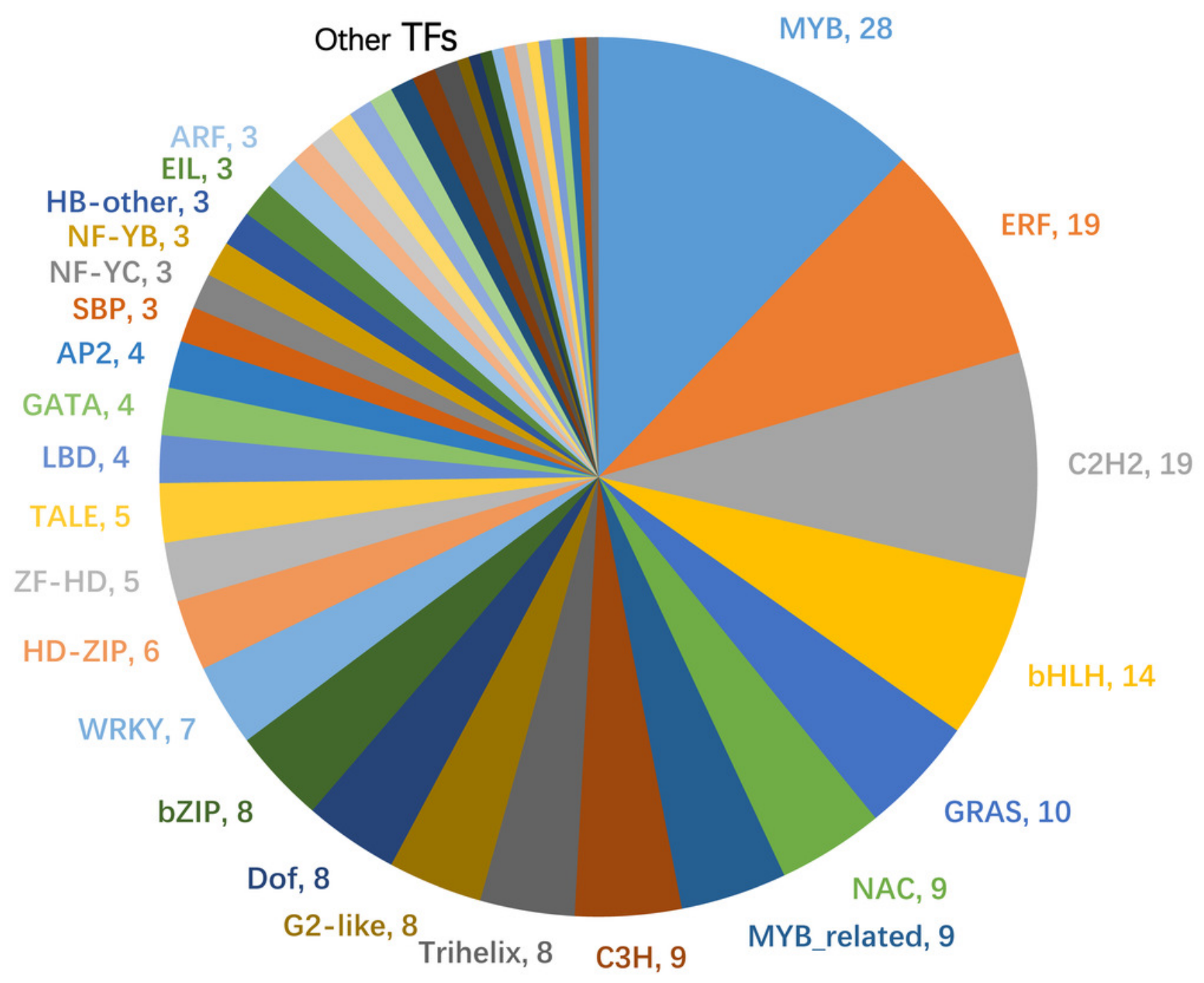




\section{Figure 9}

\section{The 23 top Kyoto Encyclopedia of Genes and Genomes (KEGG) enrichment pathways of the unigenes co-expressed with LecRLK genes.}

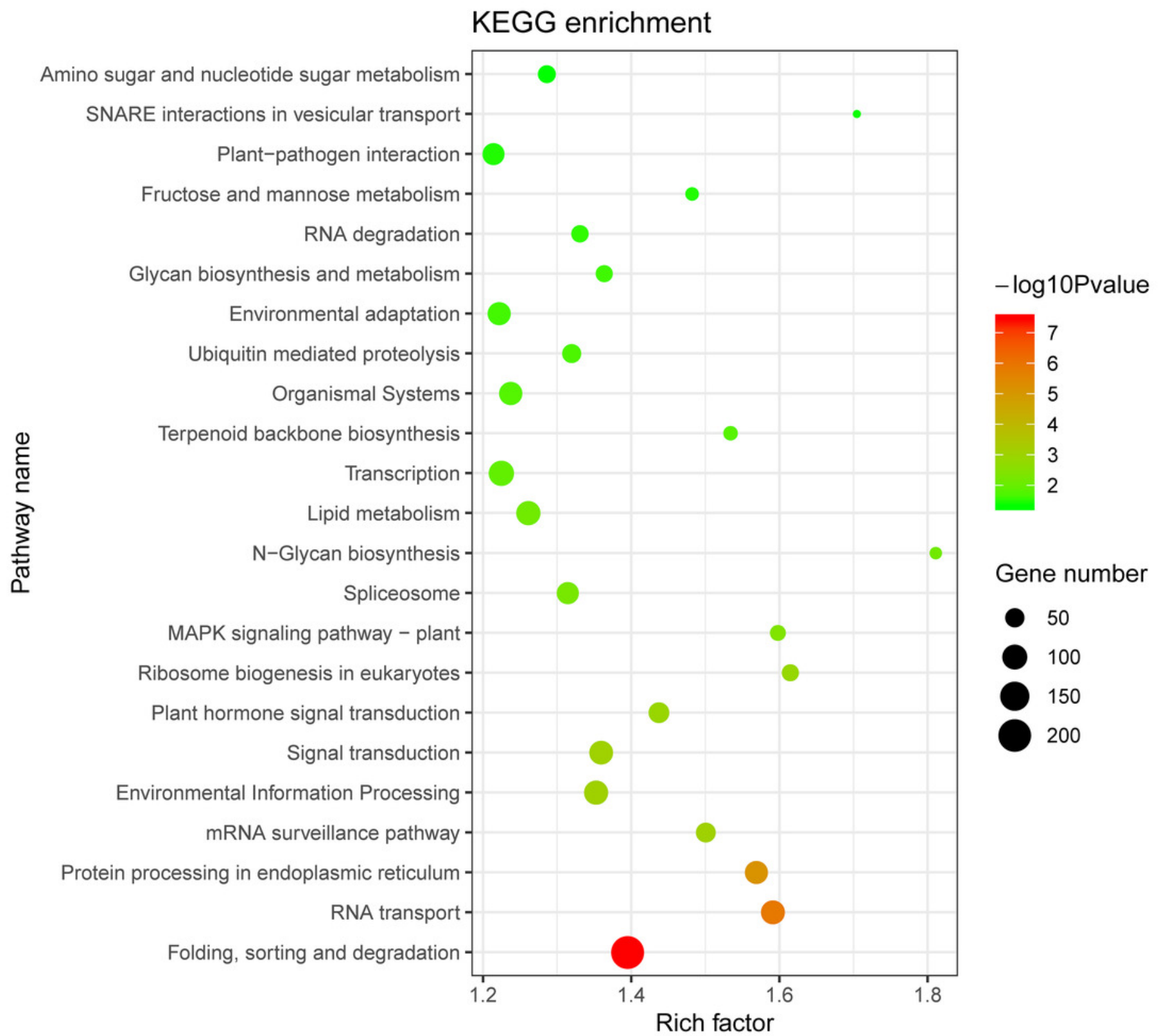




\section{Figure 10}

Expression levels of 15 LecRLK genes in T. hybrid 'Zhongshanshan' tissues.

qRT-PCR data were normalized using the APRT gene. The relative expression levels of

LecRLKs in tissues were quantified against APRT transcript levels using $2^{-\Delta \Delta C T}$. Three replicates were performed, and error bars indicate the standard deviation of three technical replicates. Values with the same letter in the same gene are not significantly different according to Duncan s multiple range tests $(P<0.05)$. 


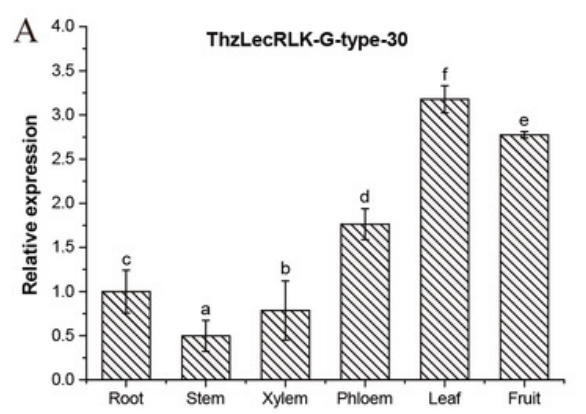

$\mathbb{\mathbb { N }}$ G type $\quad \square$ L type $\square \mathrm{C}$ type
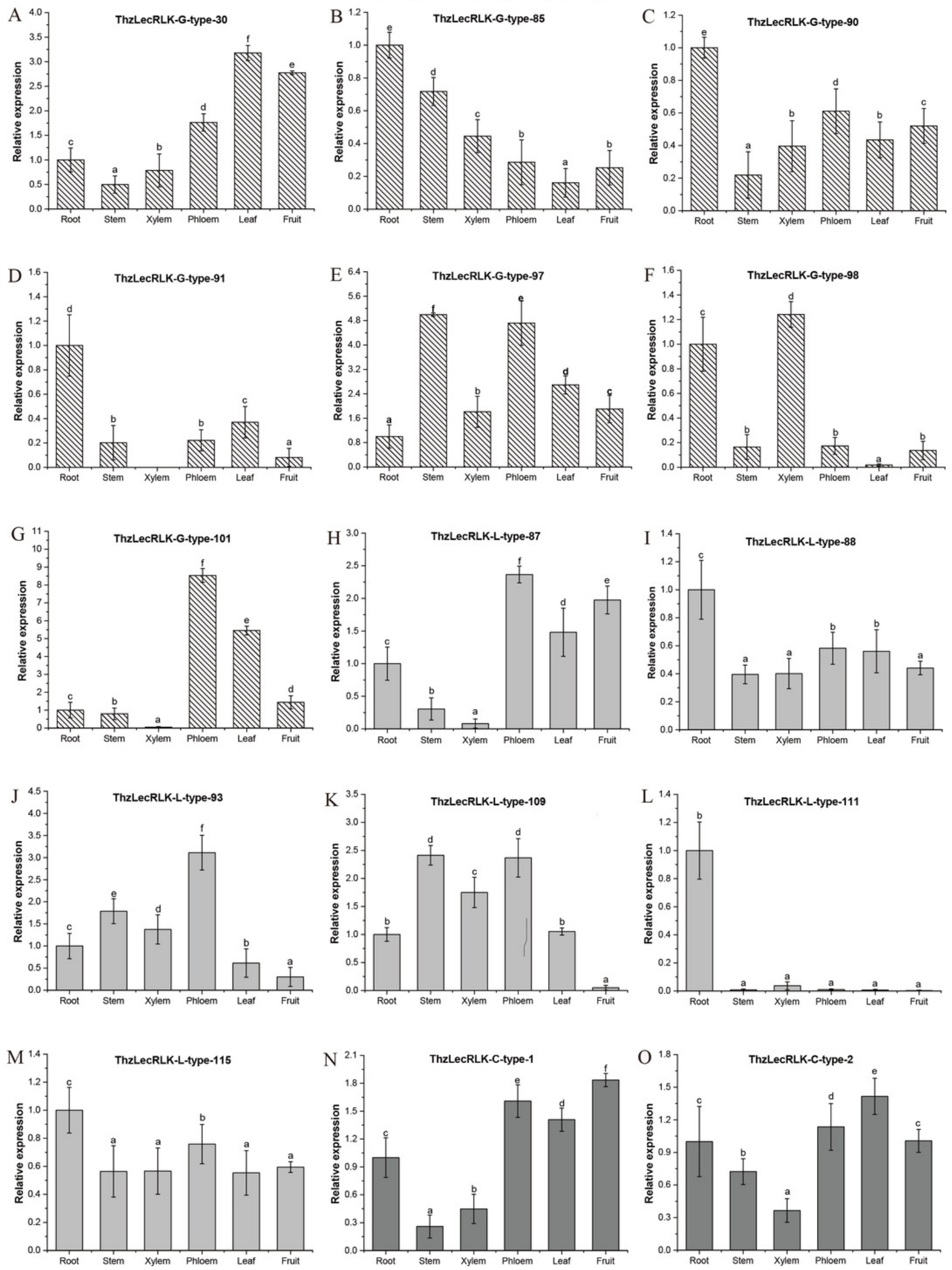


\section{Figure 11}

Expression patterns of 15 selected genes under $\mathrm{ABA}, \mathrm{SA}$, mannitol, $\mathrm{NaCl}$ and $4{ }^{\circ} \mathrm{C}$ treatment.

Different letters indicate significant differences at $\mathrm{P}<0.05$ according to Duncan's multiple range tests. qRT-PCR data were normalized using the APRT gene.Three replicates were performed, and error bars indicate the standard deviation of three technical replicates. Values with the same letter in the same gene are not significantly different according to Duncan s multiple range tests $(P<0.05)$. 


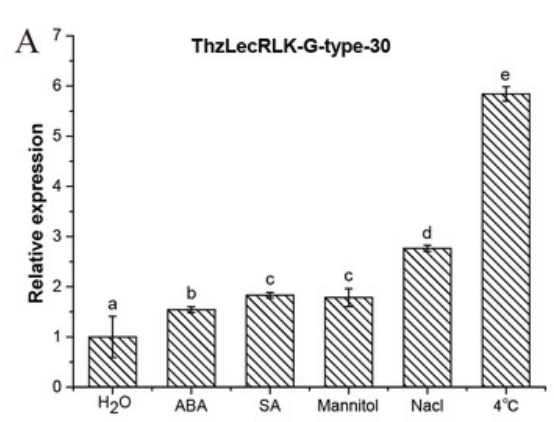

$\mathbb{\mathbb { N }}$ G type $\quad \square$ L type $\square$ C type
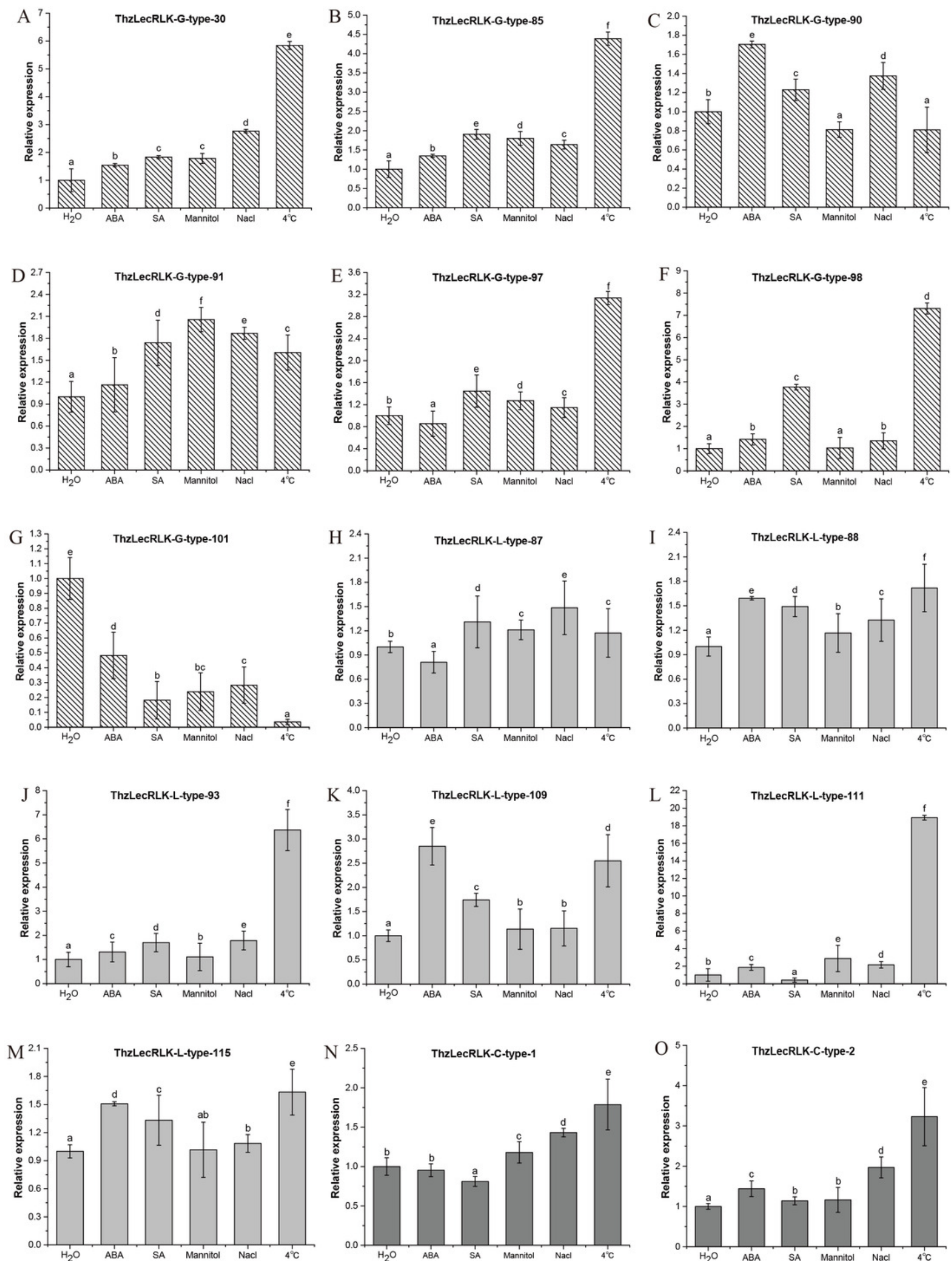


\section{Table $\mathbf{1}$ (on next page)}

Prediction of subcellular location of 297 ThzLecRLKs.

Only the consistent prediction results of the two softwares were analysised. No : The prediction results of the two softwares were not consistent. PM: plasma membrane; chlo: chloroplast; vacu: vacuole; extr: extrocytoplasmic surface; nucl: nucleus; ER: endocytoplasmic reticulum; cyto: cytoplasm; mito: mitochondrion. 
Table 1. Prediction of subcellular location of 297 ThzLecRLKs .

2

\begin{tabular}{|c|c|c|c|c|c|}
\hline type & number & percentage(\%) & type & number & Percentage(\%) \\
\hline PM & 213 & 71.7 & Extr+nucl & 1 & 0.3 \\
\hline chlo & 9 & 3 & PM+extr & 7 & 2.4 \\
\hline cyto & 6 & 2 & PM+chlo & 8 & 2.7 \\
\hline extr & 3 & 1 & PM+nucl & 2 & 0.7 \\
\hline mito & 1 & 0.3 & PM+cyto & 2 & 0.7 \\
\hline nucl & 6 & 2 & PM+mito & 1 & 0.3 \\
\hline cyto+chlo & 5 & 1.7 & PM+Vacu & 1 & 0.3 \\
\hline cyto+ER & 1 & 0.3 & No & 31 & 10.4 \\
\hline
\end{tabular}

3 1) Only the consistent prediction results of the two softwares were analysised. No: The prediction results 4 of the two softwares were not consistent.

5 2) PM: plasma membrane; chlo: chloroplast; vacu: vacuole; extr: extrocytoplasmic surface; nucl: nucleus; ER: endocytoplasmic reticulum; cyto: cytoplasm; mito: mitochondrion. 
Table 2 (on next page)

Physical and chemical properties and subcellular localization prediction of 15 ThzLeCRLKS 
1 Table 2. Physical and chemical properties and subcellular localization prediction of 15 ThzLecRLKs

\begin{tabular}{|c|c|c|c|c|c|}
\hline Unigene number & Classfication & ORF length (aa) & Theoretical pI & Molecular weight (kDa) & Location \\
\hline ThzLecRLK-G-type-101 & Group II-b & 856 & 6.71 & 95.21 & Plasma membrane \\
\hline ThzLecRLK-G-type-85 & Group II-c & 822 & 6.24 & 90.53 & Plasma membrane \\
\hline ThzLecRLK-G-type-30 & Group I & 829 & 5.44 & 93.08 & Plasma membrane \\
\hline ThzLecRLK-G-type-97 & Group V & 823 & 8.96 & 90.33 & Plasma membrane \\
\hline ThzLecRLK-G-type-90 & Group VI-a & 827 & 6.74 & 90.45 & Plasma membrane \\
\hline ThzLecRLK-G-type-91 & Group IV-a & 858 & 6.05 & 94.58 & Plasma membrane \\
\hline ThzLecRLK-L-type-88 & Group II-f & 681 & 6.44 & 76.32 & Plasma membrane \\
\hline ThzLecRLK-L-type-93 & Group III & 694 & 7.04 & 76.59 & no \\
\hline ThzLecRLK-L-type-87 & Group IV & 658 & 6.07 & 73.28 & Plasma membrane \\
\hline ThzLecRLK-L-type-111 & Group V-a & 706 & 5.15 & 78.00 & Plasma membrane \\
\hline ThzLecRLK-L-type-109 & Group VI-e & 665 & 4.97 & 74.01 & Plasma membrane \\
\hline
\end{tabular}

2 